\title{
Interactive Packaging Solutions Based on RFID Technology and Controlled Delamination Material
}

\author{
Jie Gao, Zhibo Pang, Qiang Chen and Li-Rong Zheng \\ iPack Vinn Excellence Center, School of Information and Communication Technology, Royal Institute of Technology (KTH) \\ Isafjordsgatan 39, 16440 Kista-Stockholm, Sweden \\ \{ijgao, zhibo, qiangch, lirong\}@kth.se
}

\begin{abstract}
Interactive packaging is an emerging research area in recent years. It brings people convenient and smart lives, reduces consumption of traditional packaging materials and direct or indirect labor costs as well. Being integrated in interactive packaging, Radio Frequency Identification (RFID) technology becomes one of the most proactive development enablers. In this paper, an interactive and intelligent packaging solution integrating passive RFID system and Controlled Delamination Material (CDM) is given at first. Package opening action is electrically controlled by the RFID system. CDM is primarily used in aerospace applications in the past and the conductor/adhesive joint can be easily opened by applying a little electric power on to the material. Some related works will be shown about the electrochemical characteristics of CDM in order to facilitate the system design. A demonstration system was developed and the test results have proved feasibility of the solution and shown the potential of low cost for mass production. Based on this solution, an interactive medication package for pervasive healthcare is further developed, using EPCglobal Gen2 RFID technology. It will make the medication being accessible for patient only at the prescribed dose and time, and medication taking information will be delivered as well. Such medication package will not only give unprecedented high patient compliance, but also improve the communication between patients and healthcare staffs.
\end{abstract}

Keywords - Interactive Packaging; Medicine Packages; Patient Compliance; Radio Frequency Identification (RFID); Gen2 RFID Tag; Controlled Delamination Material (CDM)

\section{INTRODUCTION}

Normally, product packaging cost is regarded as extra overhead, which means the cost can be folded into goods, but customers don't want to pay much for it. A study about the new US industry forecasts shows that the US foodservice packaging demand reaches $\$ 7.6$ billion in 2008 , and the number is $\$ 6.2$ billion in 2003 [1]. A common packaging of health and beauty product usually costs three times as much to make the content itself [2]. In addition to advertisements, packaging cost consists of raw materials, direct and indirect labor, warehousing, quality control, and related machinery, etc.

In recent years, not only packaging and forest industries, more and more companies are looking for new ways to lower their costs on packaging and logistic processes, and thus raise profit margins as well. Wal-Mart unveiled a "Packaging Scorecard" program in 2006 to improve packaging, reduce emissions and save energy [3]. Ericsson's packaging engineers created a simple calculation model that can calculate the total packaging cost and compare different packaging solutions in order to find the most effective packaging solution [4]. The emergence of interactive and intelligent packaging solutions comes to be a new way of saving the packaging cost. And these solutions are based on many high technologies, including Radio Frequency Identification (RFID) technology.

Invented in 1948, RFID is a kind of automatic identification technologies. It was fully implemented in commercial and specific applications in 1980s [5]. Comparing with barcode, RFID tags can be read at a longer distance and provide more information. The RFID system is considered as the next generation wireless communication system [6]. It is basically constituted of three main components: tag, reader or writer system, and application host. Using electromagnetic waves at radio frequencies, the reader/writer exchanges and retrieves information stored by tags attached on the objects.

RFID tags located on the objects to be identified can also be regarded as transponders. There are two types of tags: passive and active. A passive tag doesn't have power supply, and collects energy only by receiving electromagnetic radiation from reader/writer system to power up its operation [5, 7, and 8]. Although the communication distance is short, long life cycle and low cost make it considered as one of the largest commercial potential [8]. By contrast, an active tag has a battery, and can realize more complex operations and be read at a longer distance. But it is larger in size and more expensive.

A basic reader/writer system consists of a reader/writer and an antenna. In order to harvest information for RFID system applications, it is utilized to communicate with tags. The reader/writer supplies power for passive tags to make them work, and receives information from tags or writes information on tags.

For the past decade, RFID technology comes to be one of the most popular technologies and has been widely used by manufacturing industries, logistic providers, supply chain managements, retails, banks and exhibitions for the purpose of 


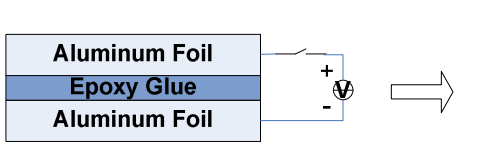

(a)

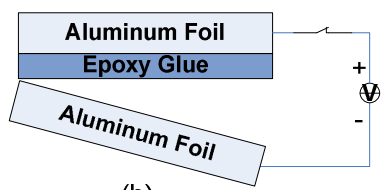

(b)
Figure 1. The structure and disbonding of CDM.

status identification, whereabouts tracking and process detection on products or animals. According to the latest RFID annual market overview from ABI Research, the total revenue earned from RFID transponders, readers, software and services will amount to more than $\$ 5.6$ billion in 2009 [9]. It is no doubt that the development trends for RFID commercial applications mainly include size miniaturization, cost reduction, energy saving, and large-scale production, etc.

Moreover, RFID technology can be combined with other technologies to expand its application scenarios. Controlled Delamination Materials (CDM) is one of them. By integrating RFID and CMD onto normal packages, interactive and intelligent packaging solutions are proposed in this paper.

A brief description about CDM structure and its basic characteristics are shown in section II. In section III, an interactive packaging solution by integrating passive RFID system and CDM onto packages is presented to show a low-cost interactive packaging solution. And the system concept, hardware details and software functions of the solution are described, including test results. In section IV, this solution is further developed and extended to pharmaceutical applications. An interactive medication package with high patient compliance for pervasive healthcare, using CDM and EPCglobal generation 2 RFID tag, is described. Section V shows the future work. And the conclusion is drawn in section VI.

\section{CONTROLLED DELAMinATION MATERIALS}

Adhesives have been used in a wide variety of traditional industries. CDM is an epoxy adhesive sandwiched between two pieces of metal substrate like aluminum foil. In order to lower the production expenses and lessen the cost of reparations, CDM was first used in aerospace applications to temporarily bond monitoring instruments to aircrafts, and permitting dismantling quickly without surface damage or marking [10]. Following the further development done by Stora Enso [11], CDM has drawn attention from the traditional packaging industry due to its characteristics of low-power and electrically controlled disbonding. It could be a new option to replace the common adhesive used to seal off a package, within the frame of developing new interactive and intelligent packaging solutions.

The typical structure of CDM is shown in Fig. 1(a). The top side and lower side are two pieces of aluminum foils. Between them, there is a thin layer of glue epoxy that has a strong

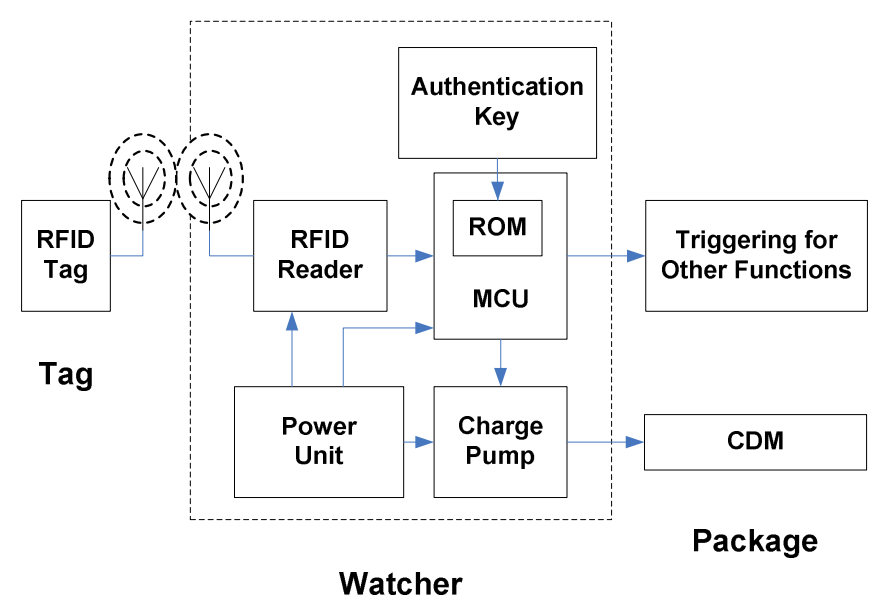

Figure 2. System concept for a smart packaging solution.

adhesion against pulling force. The strong adhesion can support a lap of shear strength in excess of $21 \mathrm{GPa}$ (3000 pounds per square inch) at ambient temperature [12], which means a bonded (unopened) CDM can afford a weight of $211 \mathrm{~kg} / \mathrm{cm}^{2}$. After a 10$50 \mathrm{~V}$ DC voltage being applied to the structure for a short period of time, the bond strength will reduced, and the adhesive will become unstuck[13, 14]. It makes CDM be easily separated by little extra force. In addition to the material properties, the total time for disbonding (opening) also depends on the power supply voltage and some environmental conditions like temperature and humidity.

In order to make CDM open, a sufficiently high voltage bias is needed to apply to both sides of the sandwiched material structure, as shown in Fig. 1(a). After applying such bias for a while, the electrochemical reaction weakens one of the interface joints between the glue epoxy and metals, and finally leads to disbonding of that interface. For the CDM structure used in this work, the aluminum foil connected to the negative side of the power supply departs from the rest part of the material after the structure being electrically biased, as shown in Fig. 1(b).

\section{AN INTERACTIVE PACKAGING SOLUTION}

\section{A. System Concept}

Sealed off with CDM, the interactive packaging can be electrically opened in a way if the specified conditions are satisfied. As shown in Fig. 2, RFID technology is used to control when and whether an opening action should take place. This lowcost solution combining CDM with a passive RFID tag is useful in fields of logistic, healthcare, security, toll system and other identification systems. The whole system includes Tag, Watcher and Package. 


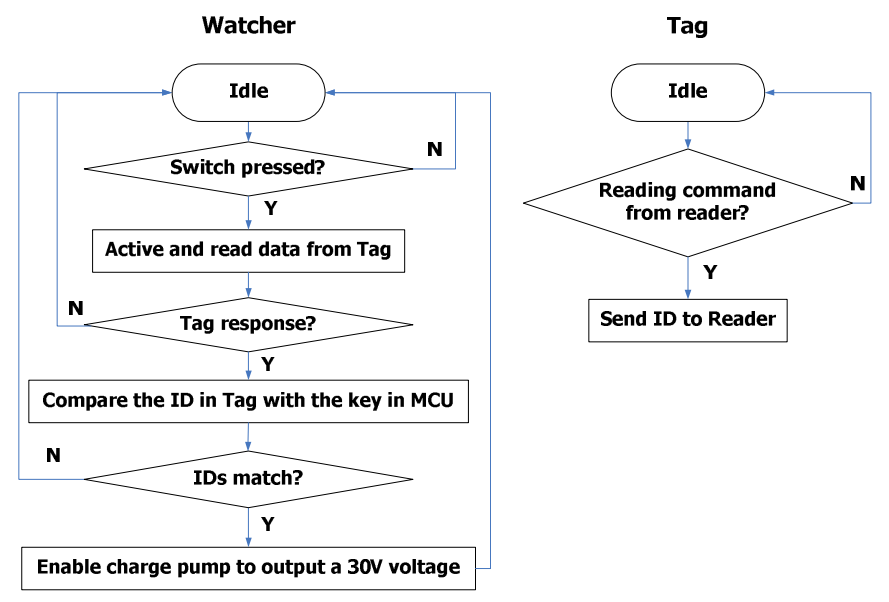

Figure 3. Flow chart of operation.

A passive RFID tag is used here. There is no extra power supply in the tag itself. An ID number is the only information on the tag. The number is unique and has been set in the factory. The tag will send data out once it captures the electromagnetic wave from the RFID reader to power up.

Five modules constitute the Watcher: an antenna, a RFID reader, a MCU, a charge pump, and a power unit. The antenna is a bridge between the tag and reader by communicating with another antenna on the tag. The reader communicates with the tag and MCU to exchange information between them, and also supplies energy to the tag by sending electromagnetic wave at radio frequency through the antenna. The MCU compares the authentication key stored on it with the ID received from RFID reader, and sends the corresponding commands for further operations. The charge pump is used to convert the output signal from the MCU to a higher voltage to drive CDM disbonding (opening action). The power unit provides the system with the power supply only when a press switch is pressed, or a touch sensor is touched respectively.

Package is connected with Watcher, and sealed off with CDM and some other external devices attached on. The output voltage from Watcher will be connected to the both sides of CDM. After being electrically biased with enough high voltage for a certain time, CDM will disbond and make Package open automatically. The external devices can provide more functionalities other than just opening, e.g. audio or video functions at the time while the package is opening.

\section{B. Functional Description}

The operational flow chart of the system function is given in Fig. 3. The firmware platform is written in $\mathrm{C}$ code and programmed to the $\mathrm{MCU}$, which is easy to reprogram through a compiler.

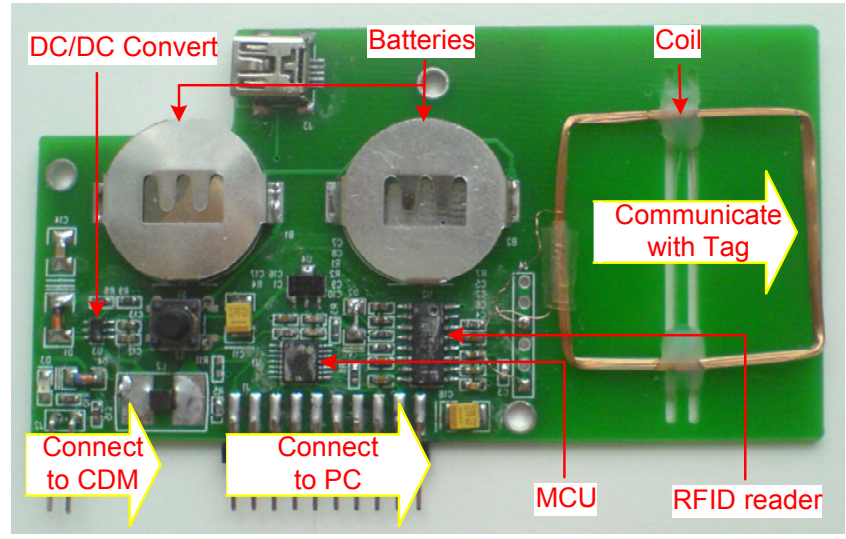

Figure 4. PCB board view of Watcher.

When Watcher is turned on by pressing the press switch or touching the touch sensor, the RFID reader starts to send signals out to find whether there are any available tags nearby or not. If a tag is presented within the reader's reading distance, it will obtain electric power by its antenna from electromagnetic wave sent by the reader. Then the tag starts to work and sends its ID number to the reader. After the reader receives an 8-bit ID number from the tag, the number is sent to the MCU and then compared by MCU with the authentication key that was pre-stored/programmed into the memory of the MCU. If these two numbers are not the same, i.e. mismatching, Watcher will be shut down after a while. If these two numbers are the same, i.e., matching, the MCU will wait a while to allow the RFID reader to read the ID again to confirm the matching. After the matching is confirmed, the MCU will enable the charge pump and later send a 30V DC output signal to Package for opening CDM sealed off the package. Meanwhile the MCU will trigger other functionalities as well, such as sound notification, etc.

\section{Hardware}

As one of the key parts of this solution, several pieces of CDM are attached on the sealing edges of the packages instead of normal sealing compound. The two metal layers of the material bonding the package connect to the voltage output end and GND of Watcher separately. It makes the package much stronger, and uneasy to open without the confirmation by a matched RFID tag.

The tag is an electronic read-only RFID card. It combines a coil antenna with a CMOS integrated circuit. The circuit is powered by the antenna placed in an electromagnetic field, and obtains its master clock from the same field via one of the antenna terminals. By turning on and off the modulation current, the chip will send back an 8-bit ID number which is coded as Manchester and stored in a factory pre-programmed memory array. For its small chip size and low power consumption, the passive RFID tag is widely used in logistics automation, anticounterfeiting, access control, and industrial transponder, etc. 


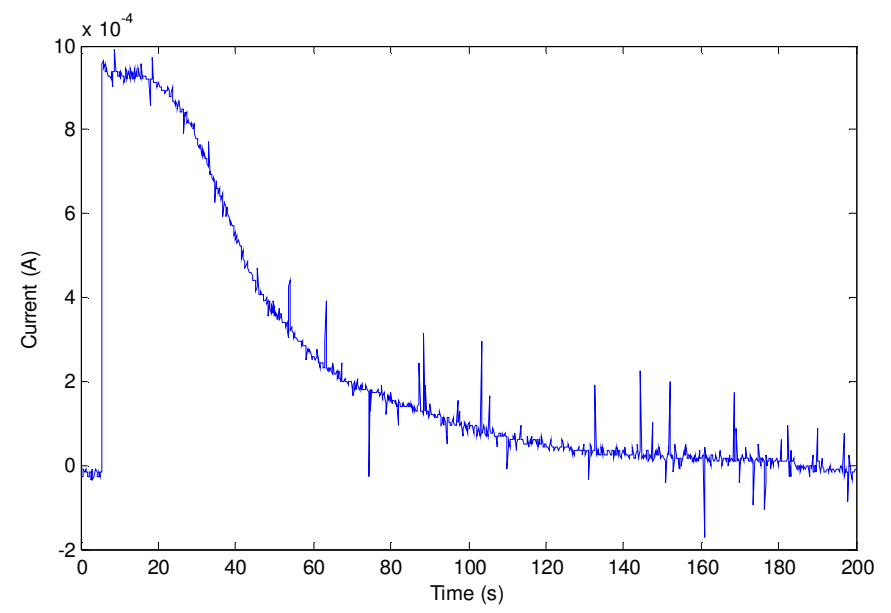

Figure 5. Current waveform on CDM at 30V DC voltage.

The main components in Watcher are a RFID reader, a MCU and a charge pump. The Watcher system in PCB board is $5.1 \mathrm{~cm} \times 9.7 \mathrm{~cm}$ in size, which is shown in Fig. 4. Since the components used in this hardware system are very common and cheap, the cost of the whole solution is low.

The RFID reader is a CMOS integrated RFID transceiver circuit. Without any external crystal oscillators, a resonant frequency which is suitable for the antenna can be obtained by the internal phase-locked loop PLL. It is used as a base station to perform antenna driving at carrier frequency, AM synchronous modulation and demodulation of the antenna signal modulation induced by the transponder, and communication with a microprocessor via simple interface. It works with a $5 \mathrm{~V}$ DC power supply and at a low frequency ranging from $100 \mathrm{kHz}$ to $150 \mathrm{kHz}$. For the small plastic package and low cost, the RFID reader is very common in car immobilizers and hand held readers.

The MCU is an 8-bit single-chip microcontroller designed for applications demanding high-integration, low-cost solutions over a wide range of requirements. It is based on a high performance processor architecture that executes instructions in 6 times the rate of standard 8051 devices. It is the core of the system to analyze data and make operation decisions.

The charge pump is a current-mode pulse-width modulation step-up DC-DC converter. The input voltage ranges from $2.5 \mathrm{~V}$ to $10 \mathrm{~V}$, and the adjustable output voltage is up to $30 \mathrm{~V}$. Because the output voltage from the MCU is only $3 \mathrm{~V}$ and the power needed to open a CDM normally should be $20 \mathrm{~V}$ or more, a DC-DC converter is necessary in this solution. If the charge pump is enabled, it will convert the input voltage from $3 \mathrm{~V}$ to $30 \mathrm{~V}$ and send it out to Package.

\section{Test Results}

\section{1) CDM DC Opening/Disbonding Behaviors}

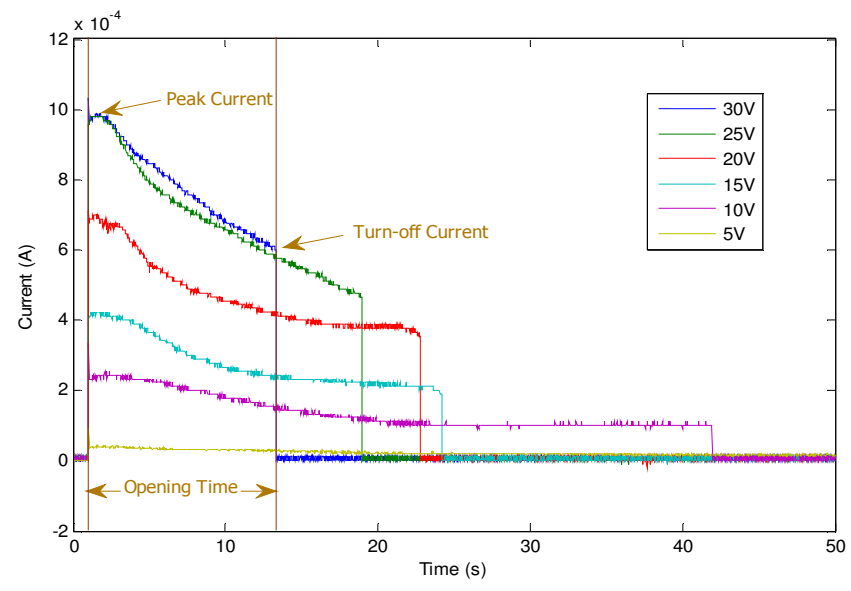

Figure 6. Current waveforms at 5-30V DC voltage.

Mentioned in many previous studies, CDM will soon open if given a potential in 10-50V DC voltage [13, 14]. And some environment parameters will affect CDM's electrochemical characteristics. The following tests are performed at a temperature round $22^{\circ}-25^{\circ}$ and a relative humidity of $30 \%-37 \%$, within the safe voltage of $36 \mathrm{~V}$ DC power supply for the human being.

When subjected to a 30V DC power supply, the waveform of the current flowing through a piece of $1 \mathrm{~cm}^{2} \mathrm{CDM}$ is given in Fig. 5.

At the beginning, while the current reaches its maximum value in a few seconds, it is nearly $0.95 \mathrm{~mA} / \mathrm{cm}^{2}$. When holding the DC voltage for about ten seconds, the current drops down substantially. After 90 seconds, the current is reduced by over $90 \%$, and the diminution will turn to slow. At this moment, the material is so weak that just a slight tough can make it peel off. But, as we can see in the last 60 seconds, without any external force, the current through the material is very small. It will infinitely close to zero but never turn to zero.

Depending on the different DC supply voltages applied, the opening time of a CDM structure greatly varies. The waveforms of the current flowing through the material under different bias voltages from 5V DC up to $30 \mathrm{~V}$ DC are shown in Fig.6. If there is no other force added, the current will never turn to zero and the material will not be separated completely as shown in Fig. 5. In order to define the delamination of the material, in this test, a certain small weight is hanged to one side of the surface for CDM.

In Fig. 6, after reaching its maximum value, the Peak Current in the whole opening process, the current slowly decreases during the following several seconds and the adhesive strength of glue becomes more and more weak. When the strength cannot afford the weight added on the material, CDM is disbonded immediately. The current at that moment is called Turn-off 


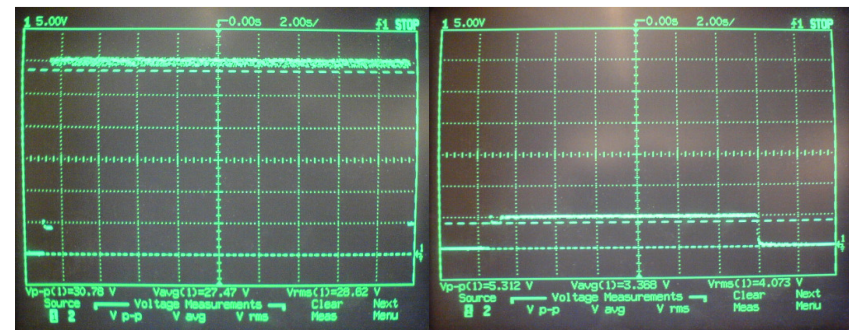

(a) Two IDs match.

(b) Two IDs do not match.

Figure 7. Voltage Pulse Generation.

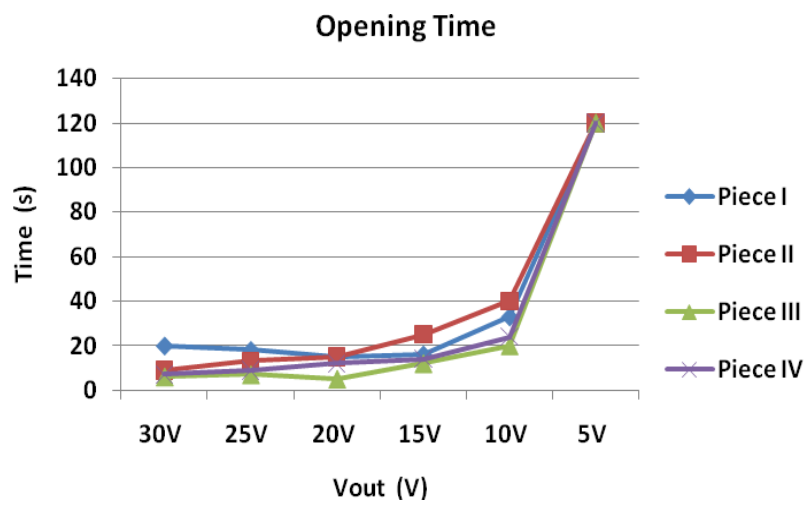

Figure 8. Opening Time at Different Power Supply.

Current. It signifies the moment that the current is cut off and the material is delaminated. And the total opening time is the time elapsed during opening process, being defined as the time interval from when the power supply is applied on the surface till when the turn-off current comes out. When the power supply is lower than 5V, CDM will not open within 2 minutes without any extra large force. When the voltage increases from $5 \mathrm{~V}$ to $30 \mathrm{~V}$, the opening time will decrease monotonically.

\section{2) Voltage Pulse Generation}

If the ID number in RFID tag is the same with the authentication key stored in MCU, the CDM sealed on the package will be opened automatically in several seconds after receiving a $30 \mathrm{~V}$ DC voltage from Watcher as Fig. 7 (a). If the two IDs are not the same, the output voltage is about $5 \mathrm{~V}$ and is too low to open Package as Fig. 7 (b). And there is a flow voltage to keep RFID reader and MCU work during the test process. It is about $4.3 \mathrm{~V}-5 \mathrm{~V}$.

\section{3) Reading Distance}

The reading distance between the tag and the package is mainly decided by the RFID system. It is about $5 \mathrm{~cm}$ in this solution, which is suitable for the application scenarios.

\section{4) Programmable Control}

The authentication key stored in the MCU can be reprogrammed by connecting to a computer. By replacing the

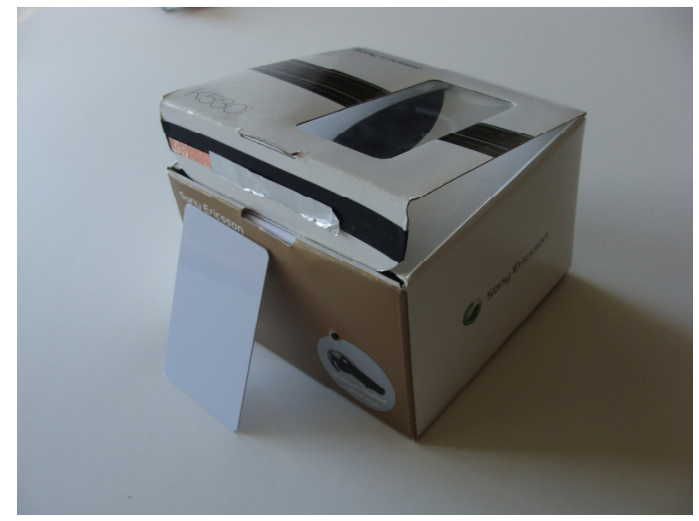

Figure 9. Automatically opening of the smart package with a RFID card.

opened CDM to a new one, it is convenient for users and manufactures to recycle the smart package.

Due to the chemical characteristics instability of the material, the time duration of the output voltage pulse from Watcher is also programmable to ensure a reliable opening for CDM.

\section{5) Opening Time of CDM}

For different pieces of the materials in different environments, the total opening time is not always the same. The test results for the opening time of 4 different pieces of CDM under different DC power supply voltages from $5 \mathrm{~V}$ to $30 \mathrm{~V}$ are shown in Fig. 8. CDM cannot be opened automatically in less than 2 minutes under a $5 \mathrm{~V}$ power supply voltage. And as a contrast, the worst case is 20 seconds at a $30 \mathrm{~V}$ DC supply voltage.

For samples with different material size but from the same piece, the difference of opening time is little. It means that the size of the material is almost unrelated with opening time. And the total opening time is much dependent on individual properties of each piece.

\section{6) Current on the Material Surface}

The current flowing through the material surface of the package in the tests decreases from $\sim 680 \mu \mathrm{A}$ to $\sim 320 \mu \mathrm{A}$ during a 19 -second voltage pulse. So as an intelligent packaging solution, such a small current on the material surface is safe to be used.

A demonstration for this interactive packaging solution is shown in Fig. 9.

\section{AN INTERACTIVE MEDICAL PACKAGE WITH High COMPLIANCE FOR PERVASIVE HEALTHCARE}

From big bottle to small unit dose package, a traditional medical package is usually utilized to protect, inform and distribute the drugs inside. However, it brings a lot of problems for pharmaceutical noncompliance, such as skipping a dose, taking too much/less or wrong drugs or at wrong time, mixing fresh pills with the old ones, prematurely discontinuing 


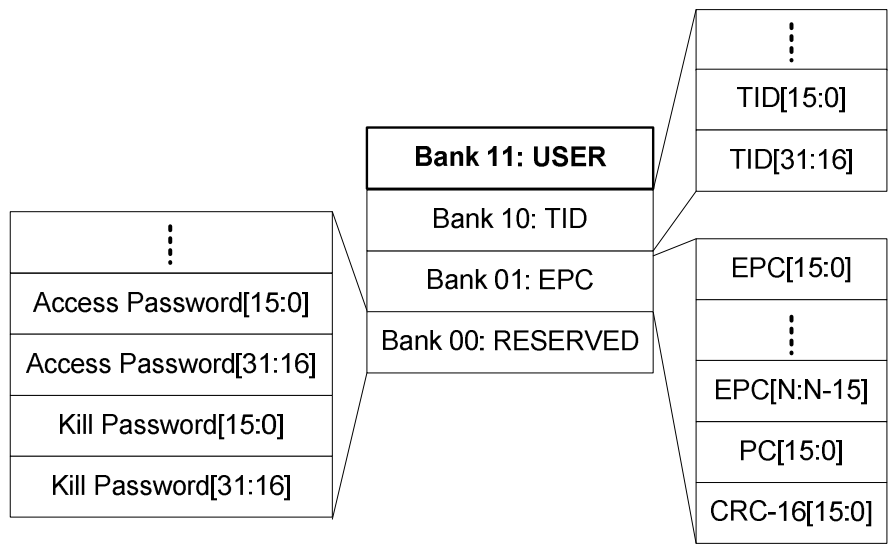

Figure 10. Logical Memory for EPC Gen2 RFID Tag[18, 19].

medication, storing medicine improperly, etc [15]. A study from the Healthcare Compliance Packaging Council (HCPC, which was established in 1990 to promote the many benefits of unit dose packaging) shows that every year in the United States only, over 125,000 death cases are attributed to pharmaceutical noncompliance, and $10 \%$ of all hospital admissions and $23 \%$ of all nursing home admissions are due to people's inability to take their medications as prescribed, which costs an estimated $\$ 180$ billion [16]. Besides these direct costs of noncompliance, the indirect costs cannot be ignored as well, such as reduction in worker productivity, more sick days and less efficient work, and premature death, etc [15]. The invention of unit dose package for medication helped to relieve these problems, and gave a certain improvement for pharmaceutical noncompliance.

Based on the aforementioned low-cost interactive packaging solution, an intelligent medical packaging solution is proposed to reach unprecedented high patient compliance for pervasive healthcare. It assists patients in taking medicine more correctly and effectively. In addition, automatically opening makes the packages smart and easy to use for seniors, and being sealed off with CDM makes the intelligent packages locked strongly enough to be child-resistant. Moreover, a more powerful generation 2 RFID tag is utilized to enhance the whole medical treatment process. Through networks, medication information will be delivered in time to improve the information exchange between patients and healthcare staffs, and enable the monitoring of patient compliance. It will be helpful for reducing the growing incidence of medical errors, decreasing the medication costs, and relieving the insufficiency of staff resources, etc.

\section{A. EPC Gen2 RFID Tag}

Being built on the original specification framework, the EPCglobal Class 1 Gen2 RFID tag has a large extensibility to many high-performance system applications. Compared with the first generation of RFID tag, Gen2 has been greatly enhanced in

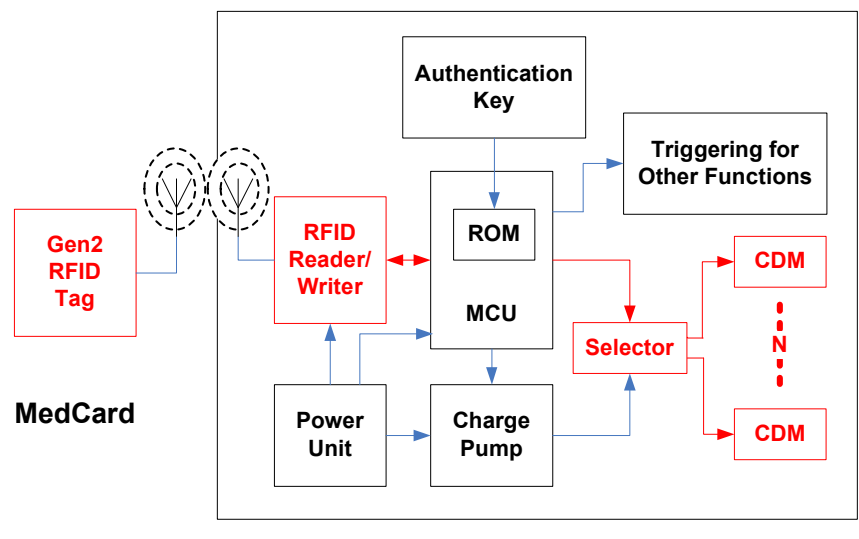

MedPackage

Figure 11. System concept.

data capacity, transmission rates, and information security, etc. The reading speed is close to four times as many as that of Gen1. Gen2 can be rewritable many times. And there are four reader "sessions" allowing parallel communication by multiple readers with one Gen2. Due to security issues, Gen2 has 32-bit lock and kill passwords. For further extensibility, the user memory depends on the manufacturer and can be as little as 0 bits to 64 bits and going as high as 2048 or more now [17]. Texas Instruments has such kinds of HF tag products for 64-bit factory programmed read only number and $2 \mathrm{M}$-bit user programmable memory.

EPCglobal Gen2 RFID tag memory is logically combined with four banks separately as shown in Fig. 10. Each bank may comprise zero or more memory words $[18,19]$. The large user memory (Bank 11) allows users to define, organize and store user data.

\section{B. System Concept}

The MedSystem is made up of three parts: MedPackage, MedCard and MedTerminal. The concept for the main parts of MedSystem is shown in Fig. 11.

\section{1) MedPackage}

In addition to the several parts used in the previous single interactive packaging solution, MedPackage has a selector and several individual packages sealed off with CDM. And the RFID reader is replaced by a RFID reader/writer. Each individual CDM sealed package could contain a given amount of a specific medicine or a specific mixture of multiple medicines.

When a MedPackage is powered up and starts to work, the RFID reader will find and read any MedCards nearby to confirm the identity of the MedCards at first. If the ID in a MedCard does not match with the authentication key kept in the MCU of the MedPackage, which means MedCard meets the wrong MedPackage, and the latter will turn off. If they match, the reader 


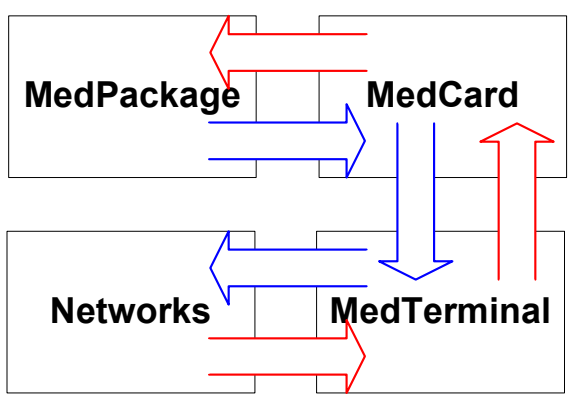

Figure 12. Information flow chart for MedSystem.

will accept all the information/prescription kept on MedCard. Then the MCU will follow the prescription and send commands to the selector to control when and how many individual packages should be opened. When the time is up, a certain amount of medicine packages will be gradually opened in turn with some visible or audible reminder functions. For the hard package sealed off with CDM, it is difficult to open without any tools for children or unauthorized opening, or if it is not the time.

Each time after a package opened, the MCU will create an opening report and send it to the RFID writer. Connected with MedCard, the report will be written on the MedCard.

\section{2) MedCard}

Being different from the basic Tag in the previous solution, MedCard is a Gen2 RFID tag that has a large programmable memory for users.

Before receiving any signals from a MedPackage, the MedCard is disabled. When it is activated by a reading signal from the RFID reader, MedCard sends out the ID number at first. If the ID is confirmed, another reading signal from the MedPackage will let MedCard send all its information to the RFID reader. Then MedCard will turn off. Once the RFID writer in MedPackage gets a report from MCU, a writing signal activates the MedCard again. Then the information stored on MedCard will be updated.

\section{3) MedTerminal}

The MedTerminal could be a networked terminal equipped with RFID reader/writer. It can be deployed in pharmacies, healthcare organization, and/or patient's home. The information from patients and healthcare organization will be processed and saved. Through the networks, the communication between patients and healthcare staffs will be highly improved.

\section{Functional Description}

The main function of MedSystem is to improve the communication between patients and healthcare staffs. Due to having a Gen2 RFID tag, MedSystem has much more data to be involved in the interaction of the whole patient treatment process. The data are valuable information, not only for the patients themselves, but also for the healthcare organization and pharmaceutical industries. The information flow chart is shown in Fig. 12.

\section{1) Open-Counterclockwise Flow}

Following the counterclockwise flow in Fig. 12, an opening route for a MedPackage is presented. The detailed standard instruction about the medicine inside will be pre-set by pharmaceutical manufactures, and it can be adjusted by hospital or pharmacy staffs. Once a patient receives his diagnosis, the MedTerminal could retrieve information through networks. The information may include the basic personal information and status, codes of the healthcare organization and doctor, case number of previous records and doctors' diagnosis, codes of the medicine, and the time and dosage to take drugs, etc. The prescription, such as the name of the medicine, when the patient should take drugs, and how many pills should be taken, is one of the most important types of the information. These data will be written on a MedCard by the RFID reader/writer of a MedTermianal. When receiving or delivering a MedCard and MedPackage(s), the patient and medical staff could check the matching between MedCard and MedPackage(s). It is effective to avoid mistakes in receiving/issuing card and/or packages. Once a matching status is confirmed, the prescription will be read and operated by the MCU in MedPackage. The right medicine packages sealed by CDM will be opened automatically following the instructions from the $\mathrm{MCU}$, which is child-resistant and senior-friendly.

\section{2) Report-Clockwise Flow}

A report route is given following the clockwise flow in Fig. 12. With the gradually opening of the packages, reports on opening success are created by MCU. They are the new records in patient's medication history. Through the communication loop from MedPackage to MedCard and then to MedTerminal, the reports will be updated in the whole MedSystem. And through the networks, the healthcare staffs could then timely retrieve these updates. They can monitor the cure plan remotely and have a decision for whether following the previous plan or doing some adjustments. If a new decision is made, the old instruction will be renewed through the networks, following again the counterclockwise flow in Fig.12. The way(s) of opening MedPackage(s) will be changed immediately. These reports will follow the whole medical treatment process, and reduce as much as possible the medical errors caused by poor communications.

\section{Functional Verification}

According to the different ID numbers getting from MedCards, MedPackage operates in different ways. The operation rules are programmable and easily controlled by the MCU in MedPackage.

In Fig. 13, two multi-output cases show the sequence of three 30V DC pulse outputs are changed with different IDs and rules. And the holding times for each output to keep the $30 \mathrm{~V} \mathrm{DC}$ voltage are changed as well, which is utilized to meet the variable needs to open the MedPackage CDM sealed. 


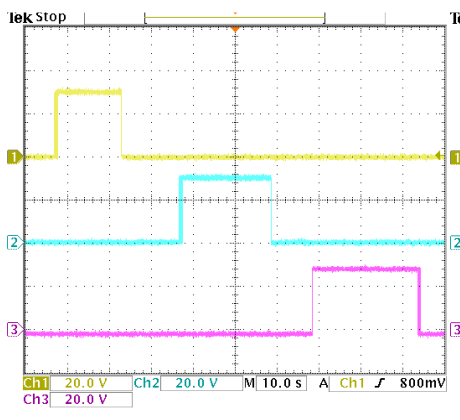

(a) Multi-output case A.

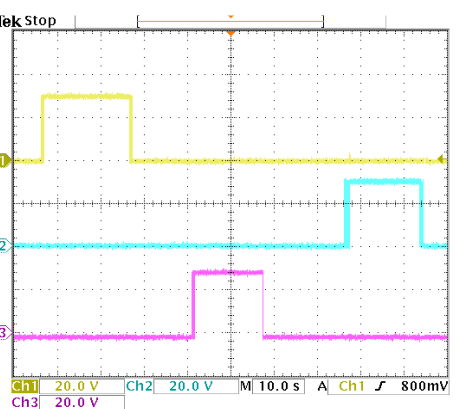

(b) Multi-output case B.
Figure 13. Programable multi-outputs waveform.

\section{FUTURE WORK}

In the future, the initial discrete component design for demonstrating the proposed interactive packaging solutions would be replaced by a SiP solution to miniaturize the system and reduce the cost. Such SiP solution might be even built on multi-layer paper based board by using printed electronics. Integrating DC/DC converter, RFID reader/writer circuits and MCU on an application specific SoC, in combination with paper battery, printed antennas and printed CDM package sealing-off, is another interesting direction towards realizing interactive packaging solutions of low cost and small size. The aim of $\mathrm{DC} / \mathrm{DC}$ converter design is to achieve a DC voltage converting from $1 \mathrm{~V} \mathrm{DC}$ to $30 \mathrm{~V}$ DC or more.

\section{CONCLUSION}

In this paper, an interactive and intelligent packaging solution integrating RFID technology and CDM is first presented. A package sealed off with CDM could be opened automatically and electrically in 20 seconds. Passive RFID technology is used to control the opening action. Test results of the demonstration have proved the feasibility of the proposed interactive packaging solution. Based on this basic solution, an interactive medical package of unprecedented high patient compliance for pervasive healthcare is suggested. Combining RFID with CDM, the medicine packages are senior-friendly, automatic to open with authorization for right dose and right time, and resistant without approval. By using a Gen2 RFID tag, a large information flow could be utilized to improve the communication between the patients and healthcare staffs.

\section{ACKNOWLEDGMENT}

This work was financially supported by Vinnova (The Swedish Governmental Agency for Innovation Systems) through the Vinn Excellence centers program at the Royal Institute of Technology (KTH).

\section{REFERENCES}

[1] The Freedonia Group, "NEW US Industry Study with forecasts to 2008 \& 2013 - Foodservice Packaging: Bulk \& Portion Control", April 2004, http://www.freedoniagroup.com/brochure/17xx/1796smwe.pdf

[2] Sean Poulter, "Product packaging can cost three times as much as what's inside", 13 July 2007, http://www.dailymail.co.uk/news/article468022/Product-packaging-cost-times-whats-inside.html

[3] Wal-Mart Stores, Inc., "Wal-Mart Unveils 'Packaging Scorecard' to Suppliers", 1 November 2006, http://walmartstores.com/FactsNews/ NewsRoom/6039.aspx

[4] Patrik De La Motte, Patrik Persson, "A packaging cost model for Ericsson AB", January 2009, http://lup.lub.lu.se/luur/download?func= downloadFile \&recordOId=1315314\&fileOId=1315931

[5] Landt J., "The history of RFID", Potentials, Vol 24, No.4, pp.8-11, Nov. 2005

[6] Dong-Liang Wu, Wing W. Y. NG, Daniel S. Yeung, Hai-Lan Ding, "A Brief Survey On Current RFID Applications", the Eighth International Conference on Machine Learning and Cybernetics, Baoding, 12-15 July 2009, p. 2230-2235

[7] M. Keskilammi, L. Syda nheimo and M. Kivikoski, "Radio Frequency Technology for Automated Manufacturing and Logistics Control. Part 1: Passive RFID Systems and the Effects of Antenna Parameters on Operational Distance", Int J Adv Manuf Technol (2003) 21:769-774

[8] Katariina Penttilä, Mikko Keskilammi, Lauri Sydänheimo, Markku Kivikoski, "Radio frequency technology for automated manufacturing and logistics control. Part 2: RFID antenna utilisation in industrial applications",Int J Adv Manuf Technol (2006) 31: 116-124

[9] Christine Gallen, “Total RFID Revenue to Exceed \$5.6 Billion in 2009, According to ABI Research", ABIrearch, http://www.abiresearch.com/press/1395

[10] EIC Laboratories, Inc., http://www.eiclabs.com.

[11] Stora Enso Oyj, http://www.storaenso.com

[12] Jeffry S. Welsh, John E Higgins, Michael D. Gilbert and Stuart F. Cogan, "Evaluation of Electrically Disbonding Adhesive Propertites for Use as Separation Systems", 44th AIAA/ASME/ASCE/AHS Structures, Structural Dynamics, and Materials Confere, 7-10 April 2003, Norfolk, Virginia.

[13] Carl-Ola Danielsson, Petronella Norberg and Lars Sandberg, "Controlled Delamination of Adhesive within Packaging and Distribution", Technical report, unpublished.

[14] Donald W. Haydon, "ElectRelease - electrically dis-bonding epoxy", Sep 2002, Volume: 22, Issue: 3, Number: 4, PageL 326-329, ISSN 0144-5154

[15] Peter Mayberry, "Increasing Pharmaceutical Compliance Through Better Packaging", 17th Annual National Symposium on Patient Compliance, May 12, 2009, Crowne Plaza Hotel, Valley Forge, Pennsylvania

[16] The Healthcare Compliance Packaging Council (HCPC), http://www.unitdose.org/ Upkar Varshney, Georgia State University, "Perasive healthcare", http://www.cis.gsu.edu/ uvarshne/papers/PervH.pdf

[17] Alien Technology Corporation, "Whitepaper - EPCglobal Class 1 Gen 2 RFID Specification," 2005, www.alientechnology.com

[18] Myunghee Son, Taewan Kim, Hyunsook Cho, "New Electronic Service Code Encoded on the UHF Class 1 Gen 2 Tag for B2C RFID Services", 9th International Conference on Telecommunications - ConTEL 2007, June 13-15, 2007, Zagreb, Croatia, Page(s):275 - 278

[19] Hung-Yu Chien, Chieh-Shian Tu, Tzong-Chen Wu, "RFID-based, Anonymous Authentication scheme", Ubiquitous, Autonomic and Trusted Computing, 2009. UIC-ATC '09. Symposia and Workshops on, 7-9 July 2009 Page(s):22 - 27 